Pacific Journal of Mathematics

METABELIAN GROUPS WITH AN IRREDUCIBLE
PROJECTIVE REPRESENTATION OF LARGE DEGREE 


\title{
METABELIAN GROUPS WITH AN IRREDUCIBLE PROJECTIVE REPRESENTATION OF LARGE DEGREE
}

F. R. DeMEYer

\begin{abstract}
The structure of a metabelian group admitting an irreducible projective complex representation whose degree is the square root of the order of the group studied.
\end{abstract}

In [7] a class of metabelian groups admitting a faithful irreducible projective representation was characterized. If $\bar{G}$ is a finite group with irreducible projective representation $T^{*}$ so that $T^{*}(\mathbf{1})^{2}=$ $[\bar{G}: 1]$ then $T^{*}$ is a faithful irreducible projective representation. Conversely, if $G$ is abelian and $T^{*}$ is a faithful irreducible projective representation then $T^{*}(1)^{2}=[\bar{G}: 1]$. We study metabelian groups $\bar{G}$ admitting an irreducible projective representation $T^{*}$ with $T^{*}(1)^{2}=$ $[\bar{G}: 1]$. Given such a group one can construct, using Schur's theory, a central extension $G$ of $\bar{G}$ with center $Z$ and an ordinary faithful irreducible representation $T$ of $G$ with $T(1)^{2}=[G: Z]$ and $T^{*}(g Z)=$ $T(g)$ for all $g \in G$ (see p. 358 of [1]). A group $G$ with an irreducible representation $T$ so that $T(1)^{2}=[G: Z]$ is called a group of central type.

Groups of central type have been studied in several places (see for example [3] and [5]). We study metabelian groups $\bar{G}$ admitting an irreducible projective complex representation whose degree is $\checkmark[\bar{G}: 1]$ by studying the associated central extension $G$ of central type using the methods of ordinary representation theory. In this note all groups are finite and all representations and characters are assumed to be taken over the complex numbers. All unexplained terminology and notation is as in [1].

I. An abelian group $G$ is said to be of symmetric type in case $G=A \times A$ for some abelian group $A$. It has been shown that an abelian group $G$ is a group of symmetric type if and only if $G$ admits a faithful irreducible projective representation [4] if and only if $G$ admits an irreducible projective representation $T^{*}$ with $T^{*}(1)^{2}=[G: 1]([2])$. For any group $H$ let $Z(H)$ denote the center of $H$.

THeOREM 1. Let $G$ be a group of central type with eenter $Z$. Assume there is a faithful irreducible character $\zeta$ on $G$ with $\zeta(1)^{2}=$ $[G: Z]$. If there is a normal subgroup $H$ of $G$ with $Z \cong H \subseteq G$ 
and both $G / H$ and $H / Z$ abelian then

(1) $H$ is a group of central type so $H / Z(H)$ is of symmetric type.

(2) There is a normal subgroup $K$ of $G$ with $H \subseteq K \leqq G$ so that $K / H$ is of symmetric type and $[G: K]=[Z(H): Z]$.

Proof. First assume $G$ is a $p$-group, $p$ a prime. By Schur's lemma $\left.\zeta\right|_{z}=\zeta(1) \phi$ for a faithful linear character $\phi$ on $Z$. Proceed by induction on $[Z(H): Z]$ to prove: If $\psi$ is an extension of $\phi$ to $Z(H)$ then $\psi^{H}=\lambda(1) \lambda$ where $\lambda(1)^{2}=[H: Z(H)]$, and thus $H$ is a group of central type with center $Z(H)$. If $[Z(H): Z]=1$ then $H$ is a group with cyclic center $Z$ such that $H / Z$ is abelian. In this case it is easy to check that the commutator map induces a nondegenerate bilinear function from $H / Z \times H / Z$ to $Z$. It follows by Proposition 3 of [2] that $H$ is of central type and $H / Z$ is of symmetric type. Moreover $\phi^{H}=\lambda(e) \lambda$ for an irreducible character $\lambda$ on $H$ with $\lambda(1)^{2}=[H: Z]$. If $Z(H) \neq Z$ let $x \in Z(H)-Z$ with $x^{p} \in Z$ and $x \cdot Z$ in the center of $G / Z$. We can find such an $x$ because $G$ is a $p$-group. Let $H_{1}=\{y \in G \mid[x, y]=e\}$. By Theorem 3 of [3], [G: $\left.H_{1}\right]=p$ and $H_{1}$ is a group of central type with center $Z\left(H_{1}\right)=Z\langle x\rangle$. If $\psi$ is an extension of $\phi$ to $Z\left(H_{1}\right)$ then $\psi^{H_{1}}=\rho(e) \rho$ where $\rho(e)^{2}=$ $\left[H_{1}: Z\left(H_{1}\right)\right]$. If $X=\operatorname{ker} \psi=\operatorname{ker} \rho$ then $X$ is a subgroup of $Z\left(H_{1}\right)$ so that $X \cap Z=\{e\}$ and $H_{1} / X$ is a group of central type with a faithful irreducible character $\rho$. Clearly $Z(H) / X \subseteq Z(H / X)$. If $h X \in Z(H / X)$ then for any $k \in H,[h, k] \in X$. But $H / Z$ is abelian so $[h, k] \in Z$, and $Z \cap X=\{e\}$. Thus $h \in Z(H)$ and $Z(H) / X=Z(H / X)$. We apply induction to the group $H_{1} / X$, and obtain $H / X$ is a group of central type with center $Z(H) / X$. Thus $H$ is a group of central type with center $Z(H)$ and $H / Z(H)$ is of symmetric type. Moreover, if $\psi$ is an extension of $\psi$ to $Z(H)$ then $\psi^{H}=\lambda(1) \lambda$ where $\lambda(1)^{2}=[H: Z(H)]$.

To prove (2) let $K=\left\{y \in G \mid \lambda^{y}=\lambda\right\}$. Since $G / H$ is abelian, $K \triangleleft$ $G$. We proceed by induction on $[Z(H): Z]$ as in the proof of (1) to show $(K, H, \lambda)$ is a fully ramified triple [5] or [6], i.e., there is an irreducible character $\rho$ on $K$ so that $\left.\rho\right|_{H}=e \lambda$ where $e^{2}=[K: H]$. This suffices by Theorem 1.1 of [5] to show $K / H$ is of symmetric type. If $Z(H)=Z$ then $K=G$ and $\rho=\zeta$ works. If $Z(H) \neq Z$ let $H_{1}, X, \psi, \lambda$ be as in the proof of (1). If $y \in G-H_{1}$ then $\lambda^{y}(x)=$ $\lambda\left(y x y^{-1}\right)=\lambda(x z)$ where $z \in Z-\{e\}$. But $\left.\lambda\right|_{Z\left(H_{1}\right)}=\lambda(e) \psi$ so $\lambda(x z)=$ $[\lambda(e) \psi(x)] \psi(z)=\lambda(x) \phi(z)=\lambda(x) \phi(z)$. Since $\phi$ is faithful, $y \notin K$ and thus $K \subseteq H_{1}$. Since $X \cap Z=\{e\}$ we can apply the same argument in $G / X$ to see $K / X$ is the intertia group of $\lambda$ in $G / H$. Apply induction to $H_{1} / X$. Then $(K / X, H / X, \lambda)$ is a fully ramified triple so $(K$, $H, \lambda)$ is fully ramified. To prove $[G: K]=[Z(H): Z]$ we have from 
Cliffords theory (p. 342 of [1]) $\left.\rho\right|_{H}=e\left(\lambda_{1}+\cdots+\lambda_{n}\right)$ where by relabeling we can let $\lambda_{1}=\lambda$ where the $\lambda_{i}$ are conjugate irreducible characters, and $n=[G: K]$. Thus $\rho(1)^{2}=[G: Z]=e^{2} \lambda(1)^{2} n^{2}=[G$ : $K][K: H][H: Z(H)][Z(H): Z]$. Since $e^{2}=[K: H], \lambda(e)^{2}=[H: Z(H)]$, and $n=[G: K]$ so we have $[G: K]=[Z(H): Z]$.

Now we show Theorem 1 is true without requiring $G$ to be a $p$-group. By the hypothesis $H$ is nilpotent so $H=\Pi_{p} H_{p}$ where $p$ are the primes such that $p \mid[G: 1]$ and the $H_{p}$ are the $p$-Sylow subgroups of $H$. Let $G_{p}$ be a Sylow $p$-subgroup of $G$, then $Z\left(G_{p}\right)=$ $G_{p} \cap Z$ and $Z\left(G_{p}\right) \subseteq H_{p} \subseteq G_{p}$. Moreover, $G_{p}$ is a group of central type whose irreducible character of large degree is faithful. (Theorem 2 of [3].) Thus $H_{p}$ is a group of central type with center $Z\left(H_{p}\right)$ and there is a normal subgroup $K_{p}$ of $G_{p}$ containing $H_{p}$ with $K_{p} / H_{p}$ of symmetric type and $\left[G_{p}: K_{p}\right]=\left[Z\left(H_{p}\right): Z\left(G_{p}\right)\right]$. By Theorem 2 of [3], $H$ is a group of central type with center $Z(H)$, and if $K$ is the normal subgroup of $G$ which is the inverse image under the natural homomorphism of $\Pi_{p} K_{p} / H_{p}$ in $G / H$ then $K / H$ is of symmetric type since each Sylow subgroup is and $[G: K]=\prod_{p}\left[G_{p}: H_{p}\right]=$ $\Pi_{p}\left[Z\left(H_{p}\right): Z\left(G_{p}\right)\right]=[Z(H): Z]$. This proves the theorem.

There is a central extension $G$ of $A_{4} \times Z_{3}$ of order $2^{3} 3^{3}$ which is of central type, $G$ is not nilpotent, yet $G$ satisfies the hypothesis of Theorem 1.

Theorem 1 does not hold if $\zeta$ is not faithful because the center of $H$ does not behave properly. However, if $G$ is a group of central type with center $Z$ and irreducible character $\zeta$ with $\zeta(e)^{2}=[G: Z]$ then the kernel $X$ of $\zeta$ is contained in $Z$ so if one is only interested in the structure of $G / Z$ it is always possible to replace $G$ by $G / X$ where $X$ is the kernel of $\zeta$ and apply Theorem 1 to $G / X$. In Theorem 1 it is not necessarily the case that $G / K \cong Z(H) / Z$ as the following example shows. Let $H$ be the elementary abelian group of order 8 generated by $x, y, z$. Let $J$ be the cyclic group of order 4 generated by $w$ and let $J$ act as a group of automorphisms of $H$ by $w(x)=y, w(y)=x z, w(z)=z$. Let $G=[H] J$ be the semidirect product. Then the center of $G$ is generated by $z$ and has order 2 . Let $\phi$ be the faithful linear character on $Z$ and extend $\phi$ to $\psi$ on $H$ by $\psi(y)=\psi(x)=1$. Then $\psi$ has 4 conjugates under $J$ so $\psi^{G}=\zeta$ has degree $=4$ and is irreducible. Thus $G$ is a group of central type, $H \triangleleft G$, and both $H$ and $G / H=J$ are abelian. Thus $H$ is of central type with center $H$, the group $K$ of Theorem 1 is $H$ and $G / H \cong J \not \equiv H / Z$. Notice, however, that in the group $G$ one can find an abelian normal subgroup $N$ such that $G / N \cong N / Z$, namely $N=\left\langle x y, w^{2} z\right\rangle$. Then $G / N \cong N / Z$ is the Klein 4-group.

Using the results in [7] it is easy to characterize the metacyclic groups $\bar{G}$ admitting an irreducible projective representation of degree 
$n=[\bar{G}: 1]^{1 / 2}$. By 1.6 and 4.1 of [7] such a group must be the semidirect product of two cyclic groups of order $n$. If $\bar{G}=\langle a, b| a^{n}=$ $\left.b^{n}=e, b a=a^{r} b\right\rangle$ is the semidirect product of a cyclic group of order $n$ with itself then by 4.4 of [7] $\bar{G}$ admits an irreducible projective representation of degree $n$ if and only if $n-1$ is the least positive integer exponent so that $1+r+\cdots+r^{n-1}=0 \bmod n$. This was pointed out to me by the referee.

We conclude by showing how to construct a group $G$ of central type with $G / Z$ metabelian. This construction will characterize metabelian groups $\bar{G}$ with an irreducible projective representation of degree $[\bar{G}: 1]^{1 / 2}$.

First it is necessary to recall some terminology and results from $\S 2$ of [6]. Let $G$ be any finite group with normal subgroup $N$ so that $G / N$ is abelian. Let $\theta$ be an irreducible character on $N$ with $\theta^{g}=\theta$ for all $g \in G$. Then $(G, N, \theta)$ is called a character triple. Let $x, y \in G$. The character $\theta$ can be extended to a character $\psi$ on the subgroup $\langle N, y\rangle$ generated by $N$ and $y$. The character $\psi^{x}$ is another extension of $\psi$ to $\langle N, y\rangle$ so $\psi^{x}=\lambda \psi$ for some linear character $\lambda$ on $\langle N, y\rangle$. Define a complex valued bilinear function on $G \times$ $G$ by the equation $\left\langle\langle x, y\rangle=\lambda\left(x y x^{-1} y^{-1}\right)\right.$. It is shown in [6] that this inner product is well defined. The bilinear function is nondegenerate if $\{x \in G \mid\langle x, y\rangle=1$ for all $y \in G\}=N$. By 2.7 of [6] the triple $(G$, $N, \theta)$ is fully ramified if and only if the bilinear function defined above is nondegenerate.

Begin with an abelian group $Z_{1}$ and a cyclic subgroup $Z$. Let $\phi$ be a linear character on $Z_{1}$ such that $\left.\phi\right|_{z}$ is faithful. Let $H$ be an abelian group of symmetric type and let $\gamma: H \times H \rightarrow Z$ be a nondegenerate bilinear function. Such a function exists if and only if $\exp (H)$ divides [ $Z: 1]$. Let $H$ be any central extension of $Z$ by $H$ with factor set $\gamma$. Then $\left(H, Z_{1}, \phi\right)$ is a fully ramified triple and thus is a group of central type with center $Z(H)=Z_{1}$. Also $\phi^{H}=$ $\lambda(e) \lambda$ for an irreducible character $\lambda$ on $H$ with $\lambda(e)^{2}=[H: Z(H)]$. Next let $\bar{K}$ be an abelian group of symmetric type. Let $\alpha: \bar{K} \rightarrow$ Aut $(H)$ so that $\phi^{\alpha(x)}=\phi$ for all $x \in \bar{K}$ and $Z \subseteq\left\{y \in Z_{1} \mid \alpha(x)(y)=y\right.$ for all $x \in \bar{K}$. Note that $\phi^{\alpha(x)}=\phi$ if and only if $\lambda^{\alpha(x)}=\lambda$ since $\lambda$ vanishes off $Z_{1}$ and $Z_{1}$ is characteristic in $H$. Let $K$ be one of the normal extensions of $H$ by $\bar{K}$ determined by $\alpha$. Then $(K, H, \lambda)$ is a character triple and $(K, H, \lambda)$ is fully ramified if and only if the form $《$,$\rangle defined above is nondegenerate. In this case \lambda^{K}=\rho(e) \rho$ where $\rho$ is irreducible on $K$ and $\rho(e)^{2}=[K: Z]$. We choose a $K$ so that $(K, H, \lambda)$ is fully ramified. Finally let $C$ be an abelian group with $[C: 1]=\left[Z_{1}: Z\right]$ and let $\beta: C \rightarrow \operatorname{Aut}(K)$ such that $\phi^{\beta(c)} \neq \phi$ for all $c \neq e$ and $Z \subseteq\{y \in K \mid \beta(c)(y)=y$ for all $c \in C\}$. Let $G$ be any normal extension of $K$ by $C$ determined by $\beta$ with $G / H$ abelian. 
Then $\rho^{G}=\zeta$ is irreducible on $G$ since $\rho$ is distinct from all its conjugates under $C$ and $\zeta(e)^{2}=[G: C]^{2} \rho(e)^{2}=[G: Z]$. Thus $G$ is a group of central type satisfying the hypothesis of Theorem 1 and an examination of the proof of Theorem 1 shows every group satisfying the hypothesis of Theorem 1 is constructed in this way.

\section{REFERENCES}

1. C. W. Curtis and I. Reiner, Representation Theory of Finite Groups and Associative Algebras, Interscience, New York, 1962, 2nd ed., 1966.

2. F. R. DeMeyer, Galois theory in separable algebras over commutative rings, Ill. J. Math., 10 (1966), 287-295.

3. F. R. DeMeyer and G. J. Janusz, Finite groups with an irreducible representation of large Degree, Math. Zeit., 108 (1979), 145-153.

4. R. Frucht, Über die Darstellung endlicher abelscher Gruppen durch Kollineationen, J. Reine Angew. Math., 166 (1931), 16-29.

5. S. M. Gagola, Characters fully ramified over a normal subgroup, Pacific J. Math., 55 (1974), 107-126.

6. I. M. Isaacs, Characters of solvable and symplectic groups, Amer. J. Math., 95 (1973), 594-635.

7. H. N. Ng, Faithful irreducible projective representations of metabelian groups, J. Algebra, 38 (1976), 8-28.

Received June 9, 1977 and in revised form November 18, 1977.

Colorado State University

FORT COLLINS, CO 80523 



\section{PACIFIC JOURNAL OF MATHEMATICS}

\section{EDITORS}

RICHARD ARENS (Managing Editor)

University of California

Los Angeles, CA 90024

Charles W. Curtis

University of Oregon

Eugene, OR 97403

C. C. MOORE

University of California

Berkeley, CA 94720

\section{J. DugundJI}

Department of Mathematics

University of Southern California

Los Angeles, CA 90007

R. FinN and J. Milgram

Stanford University

Stanford, CA 94305

\section{ASSOCIATE EDITORS}
E. F. BECKENBACH
B. H. NeumanN
F. WOLF
K. YosHIDA

\section{SUPPORTING INSTITUTIONS}

\author{
UNIVERSITY OF BRITISH COLUMBIA \\ CALIFORNIA INSTITUTE OF TECHNOLOGY \\ UNIVERSITY OF CALIFORNIA \\ MONTANA STATE UNIVERSITY \\ UNIVERSITY OF NEVADA, RENO \\ NEW MEXICO STATE UNIVERSITY \\ OREGON STATE UNIVERSITY \\ UNIVERSITY OF OREGON
}

\author{
UNIVERSITY OF SOUTHERN CALIFORNIA \\ STANFORD UNIVERSITY \\ UNIVERSITY OF HAWAII \\ UNIVERSITY OF TOKYO \\ UNIVERSITY OF UTAH \\ WASHINGTON STATE UNIVERSITY \\ UNIVERSITY OF WASHINGTON
}

The Supporting Institutions listed above contribute to the cost of publication of this Journal, but they are not owners or publishers and have no responsibility for its content or policies.

Mathematical papers intended for publication in the Pacific Journal of Mathematics should be in typed form or offset-reproduced, (not dittoed), double spaced with large margins. Please do not use built up fractions in the text of the manuscript. However, you may use them in the displayed equations. Underline Greek letters in red, German in green, and script in blue. The first paragraph or two must be capable of being used separately as a synopsis of the entire paper. Items of the bibliography should not be cited there unless absolutely necessary, in which case they must be identified by author and journal, rather than by item number. Manuscripts, in triplicate, may be sent to any one of the editors. Please classify according to the scheme of Math. Reviews, Index to Vol. 39. All other communications should be addressed to the managing editor, or Elaine Barth, University of California, Los Angeles, California, 90024.

50 reprints to each author are provided free for each article, only if page charges have been substantially paid. Additional copies may be obtained at cost in multiples of 50 .

The Pacific Journal of Mathematics is issued monthly as of January 1966. Regular subscription rate: $\$ 72.00$ a year (6 Vols., 12 issues). Special rate: $\$ 36.00$ a year to individual members of supporting institutions.

Subscriptions, orders for numbers issued in the last three calendar years, and changes of address should be sent to Pacific Journal of Mathematics, 103 Highland Boulevard, Berkeley, California, 94708. Older back numbers obtainable from Kraus Periodicals Co., Route 100, Millwood, NY 10546.

PUBLISHED BY PACIFIC JOURNAL OF MATHEMATICS, A NON-PROFIT CORPORATION

Printed at Kokusai Bunken Insatsusha (International Academic Printing Co., Ltd.). 8-8, 3-chome, Takadanobaba, Shinjuku-ku, Tokyo 160, Japan.

Copyright (C) 1978 by Pacific Journal of Mathematics Manufactured and first issued in Japan 


\section{Pacific Journal of Mathematics}

\section{Vol. 76, No. $2 \quad$ December, 1978}

Stephanie Brewster Brewer Taylor Alexander, Local and global convexity in complete Riemannian manifolds ...........................

Claudi Alsina i Català, On countable products and algebraic convexifications of probabilistic metric spaces ...............................

Joel David Berman and George Grätzer, Uniform representations of

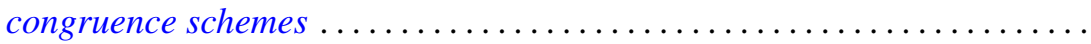

Ajit Kaur Chilana and Kenneth Allen Ross, Spectral synthesis in

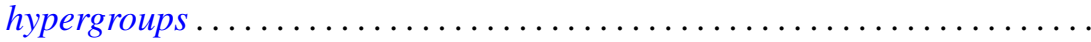

David Mordecai Cohen and Howard Leonard Resnikoff, Hermitian quadratic forms and Hermitian modular forms . .........................

Frank Rimi DeMeyer, Metabelian groups with an irreducible projective

representation of large degree .............................

Robert Ellis, The Furstenberg structure theorem .....................

Heinz W. Engl, Random fixed point theorems for multivalued mappings .......

William Andrew Ettling, On arc length sharpenings ..................

Kent Ralph Fuller and Joel K. Haack, Rings with quivers that are trees........

Kenneth R. Goodearl, Centers of regular self-injective rings ...............

John Gregory, Numerical algorithms for oscillation vectors of second order

differential equations including the Euler-Lagrange equation for

symmetric tridiagonal matrices.

Branko Grünbaum and Geoffrey Shephard, Isotoxal tilings

Myron Stanley Henry and Kenneth Leroy Wiggins, Applications of

approximation theory to differential equations with deviating

arguments

Mark Jungerman, The non-orientable genus of the n-cube .

Robert Richard Kallman, Only trivial Borel measures on $S_{\infty}$ are

quasi-invariant under automorphisms ................

Joyce Longman and Michael Rich, Scalar dependent algebras in the

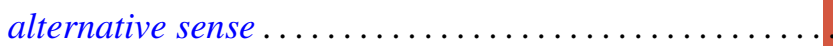

Richard A. Mollin, The Schur group of a field of characteristic zero ........ 471

David Pokrass, Some radical properties of rings with $(a, b, c)=(c, a, b) \ldots 479$

Margaret Shay and Paul Ruel Young, Characterizing the orders changed by

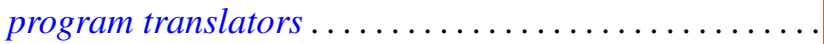

Jerrold Norman Siegel, On the structure of $B_{\infty}(F), F$ a stable space...

Surjeet Singh, (hnp)-rings over which every module admits a basic

submodule...

A. K. Snyder, Universal interpolating sets and the Nevanlinna-Pick property in

Banach spaces of functions...

Jeffrey D. Vaaler, On the metric theory of Diophantine approximation ... 